Pacific Journal of Mathematics

THE ENVELOPES OF HOLOMORPHY OF TUBE DOMAINS IN 


\title{
THE ENVELOPES OF HOLOMORPHY OF TUBE DOMAINS IN INFINITE DIMENSIONAL BANACH SPACES
}

\author{
H. J. BREMERMANN
}

1. Introduction. Let $B$ be a Banach space with the strong topology generated by the norm. An open and connected set is called a domain. Let $f$ be a complex valued functional defined in a domain $D$ of a complex Banach space $B_{c}$. Let $L$ be a finite dimensional translated complex linear subspace of $B_{c}: L=\left\{z \mid z=z_{0}+\tau_{1} a_{1}+\cdots \tau_{n} a_{n}\right\}$ where $z_{0}, a_{1}, \cdots, a_{n}$ are fixed elements $\tau_{1}, \cdots, \tau_{n}$ complex parameters. (In the following we will call $L$ an "affine subspace"). $f$ is called " $G$-holomorphic" (=Gâteauxholomorphic) if and only if the restriction of $f$ to the intersection $D \cap L$ of $D$ with any finite dimensional affine subspace $L$ of $B_{c}$ is holomorphic (in the ordinary sense). (Compare Hille-Phillips [7], Soeder [9], Bremer$\operatorname{mann}[5]$.

A functional that is $G$-holomorphic and locally bounded is called " $F$-holomorphic" (Fréchet-holomorphic). For finite dimension the notions (ordinary) "holomorphic function" and " $G$ - and $F$-holomorphic functional" coincide. (The theory of holomorphic functionals in finite dimensional Banach spaces is equivalent to the theory of $n$ complex variables.) For infinite dimension, in general, there exist already linear (and hence $G$-holomorphic) functionals that are not locally bounded (and hence not $F$-holomorphic).

In Bremermann [5] it has been shown that the phenomenon of "simultaneous holomorphic continuation," well known for $n$ complex variables, persists for infinite dimension even for the very general $G$-holomorphic functionals: There exist domains such that all $G$-holomorphic functionals can be continued into a larger domain.

A domain for which a $G$-holomorphic functional exists that cannot be continued is called (in analogy to finite dimension) a "domain of G-holomorphy." In Bremermann [5] it has been shown that a domain of $G$-holomorphy is "pseudo-convex" (in a sense which is a natural extension from finite dimension).

We will apply these notions in the following to infinite dimensional tube domains and moreover we will show that it is possible to define and to determine the envelope of holomorphy of tube domains.

Finite dimensional tube domains and their envelopes of holomorphy have been studied by Bochner [1], Bochner-Martin [2], Hitotumatu [8], and Bremermann [3], [4]. It has been shown that a tube domain is pseudo-convex if and only if it is convex, and that the envelope of

Received January 11, 1960. This research has in part been supported by the Office of Naval Research under Contract Nonr 447 (17). 
holomorphy of any tube domain is its convex envelope. The former property has been extended to infinite dimension in [5]. We extend here the latter. To the author's knowledge this is the first time that the envelope of holomorphy of a class of infinite dimensional domains has been determined. At the same time the proof given in the following is simpler than some previous proofs for finite dimension.

2. Tube domains, envelopes of holomorphy, Let $B_{c}$ be a complex Banach space that is split into a real and imaginary part, such that every $z \in B_{c}$ is written

$$
z=x+i y \text {, where } x \in B_{r}, y \in B_{r},
$$

where $B$, is a real Banach space. Then a domain $T_{X}$ is called a tube domain with basis $X$ if and only if it is of the form $T_{X}=\{z \mid x \in X, y$ arbitrary\}, where $X$ is a domain in $B_{r}$.

Obviously, $T_{X}$ is convex if and only if $X$ is convex, and $X$ is convex if and only if the intersection of $X$ with every finite dimensional affine subspace $L_{r}$ of $B_{r}$ is convex. $\left(L_{r}=\left\{x \mid x=x_{0}+t_{1} a_{1}+\cdots, t_{n} a_{n}\right\}\right.$, where $x_{0}, a_{1}, \cdots, a_{n}$ are fixed elements in $B_{,}$, and $t_{1}, \cdots, t_{n}$ real parameters).

It is somewhat difficult to define the envelope of holomorphy for arbitrary domains. Already for finite dimension it may not be schlicht. (Comp. [3], [6]). However, for finite dimension the following is true. Let $D$ be a given domain. Suppose we have a domain $E(D)$ with the following properties:

(I) Every function holomorphic in $D$ can be continued as a (singlevalued) holomorphic function to $E(D)$.

(II) To every finite boundary point $z_{0}$ of $E(D)$ there exists a function that is holomorphic throughout $E(D)$ and is singular at $z_{0}$. If $E(D)$ has these properties, then $E(D)$ is the envelope of holomorphy of $D$.

Analogously, if we have an infinite dimensional domain $D$ and a domain $E(D)$ with the properties (I) and (II) (with respect to G-holomorphic functionals), then we call $E(D)$ the envelope of $G$-holomorphy of $D$.

3. Proof of the main theorem. Let $T_{X}$ be a tube domain that is not convex. Then, there exists an affine subspace

$$
L_{r}=\left\{x \mid x=x_{0}+t_{1} a_{1}+\cdots t_{n} a_{n}\right\}
$$

$\left(x_{0}, a_{1}, \cdots, a_{n} \in B_{r}, t_{1}, \cdots, t_{n}\right.$ real parameters $)$ such that $X \cap L_{r}$ is not convex.

Now it would be possible that $X \cap L_{r}$ is not connected and each connected component is convex (for instance if $L_{r}$ is one-dimensional). 
If $X$ is not convex, then there exist two points $x_{1}$ and $x_{2}$ that cannot be connected by a straight line segment in $X$. However, $X$ is connected, and even arcwise connected. Hence we can connect $x_{1}$ and $x_{2}$ by an arc in $X$, and even by a "polygon" that is by finitely many straight line segments. The polygonal arc spans a finite dimensional affine subspace $L_{r}$ and the connected component of $L_{r} \cap X$ that contains $x_{1}$ and $x_{2}$ is not convex since $x_{1}$ and $x_{2}$ cannot be connected by a straight line.

Thus $L_{r} \cap X$ has a connected component that is not convex. Hence there exists a point $x_{3}$ on the boundary of $L_{r} \cap X$ and a line segment $s$ containing $x_{3}$ such that $s$ is locally a supporting line segment of the complement of $L_{r} \cap X$. In particular, $x_{3}$ and $s$ can be chosen such that in a neighborhood of $x_{3}$ the line segment $s$ has with the boundary $\partial\left(X \cap L_{r}\right)$ only the point $x_{3}$ in common.

Let the equation of the line containing $s$ be

$$
s=\left\{x \mid x=x_{3}+b t\right\},
$$

where $b$ is a fixed element in $B_{r}, t$ a real parameter. Let $b$ be normalized such that $\|b\|=1$. This real line lies in the analytic plane:

$$
A=\left\{z \mid z=x_{3}+b \tau\right\},
$$

where $\tau$ is a complex parameter.

Let $S_{\rho}$ be a disc on $A$ with center at $x_{3}$, radius $\rho$ :

$$
S_{\rho}=\left\{z\left|z=x_{3}+b \tau,\right| \tau \mid<\rho\right\} .
$$

If $\rho$ is small enough, then $S_{\rho}$ will lie entirely in $T_{X}$, except for the points

$$
\left\{z\left|z=x_{3}+i b t,\right| t \mid<\rho, t \text { real }\right\} .
$$

We now apply the following lemma (which is an immediate consequence of the "fundamental Lemma" 3.1 (and 3.2) of [5] and Theorem 6.3 of [6]).

To formulate the lemma we need the distance function $d_{D}(z)$ which is defined as follows: Given a domain $D$, then

$$
d_{D}(z)=\sup r \ni\left\{z^{\prime} \mid\left\|z-z^{\prime}\right\|<r\right\} \subset D,
$$

in other words $d_{D}(z)$ is the distance of the points $z$ from the boundary of $D$, measured in the norm of $B_{c}$.

LEMmA. Let $h(z)$ be the solution of the boundary value problem

$$
\begin{aligned}
& h(\tau)=\log d_{T_{X}}\left(x_{3}+b \tau\right) \text { for }|\tau|=\rho, \\
& h(\tau) \text { harmonic for }|\tau|<\rho .
\end{aligned}
$$


Then any function that is G-holomorphic in $T_{x}$ can be continued Gholomorphically into the point set:

$$
C=\left\{z\left|z^{\prime}=x_{3}+\tau b,\right| \tau \mid<\rho,\left\|z-z^{\prime}\right\|<e^{h(\tau)}\right\} .
$$

(We note that even though $\log d_{T_{X}}(x)$ becomes infinite at the two points $z=x_{3} \pm i \rho b$, the solution of the boundary value problem exists and is finite for all $|\tau|<\rho$ ).

The pointset $C$ is a neighborhood of the point $z=x_{3}$. In particular it contains the points $\left\|z-x_{3}\right\|<e^{h(0)}$, and $e^{h(0)} \neq 0$. This continuation procedure can be repeated at any point $z=x_{3}+i y$, where $y$ is arbitrary. We always get the same neighborhood, independently of $y$, because the function $d_{T_{X}}\left(x_{3}+i y\right)$ and hence $h$ does not depend upon $y$. Hence any function $G$-holomorphic in $T_{X}$ can not only be continued into a larger domain but into a larger tube domain $T_{X^{\prime}}$, that means $X \subset X^{\prime}, X \neq X^{\prime}$.

We have to observe however one difficulty: If the intersection $X \cap\left\{X \mid\left\|x-x_{3}\right\|<e^{h(0)}\right\}$ consists of more than one component, then continuation into $T_{X^{\prime}}$, with $X^{\prime}=X \cup\left\{x \mid\left\|x-x_{3}\right\|<e^{h(0)}\right\}$ could possibly be such that the continued function would no longer be single-valued in $T_{X^{\prime}}$. In order to keep the continuation single-valued we remove from $X^{\prime}$ all components of $X \cap\left\{x \mid\left\|x-x_{3}\right\|<e^{h(0)}\right\}$ except the one that intersects $S_{\rho}$. In this way the continuation remains single-valued.

Thus we have the result: If $T_{X}$ is a tube domain such that $X$ is not convex, then any function that is $G$-holomorphic can be continued $G$-holomorphically (and single-valued) into a larger tube domain with basis $X^{\prime}$. Then we can apply the same result to $T_{X^{\prime}}$, and obviously the process can be iterated as long as the enlarged tube is not yet convex. Thus we have proved:

Given a tube domain $T_{X}$, then any function that is G-holomorphic in $T_{X}$ can be continued G-holomorphically into the convex envelope of $T_{X}$.

(The convex envelope of $T_{X}$ equals $T_{C(X)}$, where $C(X)$ is the convex envelope of $X$.)

On the other hand there exists to every boundary point $z_{0}$ of $T_{C(X)}$ a supporting affine subspace of $B_{c}$ and a linear functional $l(z)$ that becomes zero exactly on the affine subspace. (This is an immediate consequence of the Hahn-Banach theorem.) The functional $1 / l(z)$ is then $G$-holomorphic in $T_{C(X)}$ and becomes singular at $z_{0}$. Hence we have shown:

To every boundary point $z_{0}$ of a convex tube domain there exists a functional that is $G$-holomorphic in the domain and singular at $z_{0}$. The two statements combined give:

THeOREM. Let $T_{X}$ be a tube domain in a complex Banach space (of arbitrary dimension). Then the envelope of G-holomorphy of $T_{X}$ is the convex envelope of $T_{X}$, which equals $T_{C(X)}$, where $C(X)$ is the convex envelope of $X$. 


\section{BIBLIOGRAPHY}

1. S. Bochner, A theorem on analytic continuation of functions in several variables, Annals of Math., 39, (1938), 14-19.

2. S. Bochner and W. T. Martin, Several Complex Variables, Princeton, 1948.

3. H. J. Bremermann, Die Holomorphiehüllen der Tuben-und Halbtubengebiete, Math. Annalen, 127 (1954), 406-423.

4. - Complex convexity, Trans. Amer. Math. Soc., 82 (1956), 17-51.

5. - Holomorphic functionals and complex convexity in Banach spaces, Pacific J. Math., 7 (1957), 811-831. (Errata in vol. 7 at the end).

6. - Construction of the envelopes of holomorphy of arbitrary domains, Revista Mat. Hisp. Amer. 17 (1957), 1-26.

7. E. Hille and R. S. Phillips, Functional analysis and semi-groups, Amer. Math. Soc. Publ., Revised Ed., New York, 1957.

8. S. Hitotumatu, Note on the envelope of regularity of a tube domain, Proc. Japan. Acad. 26 (1950), 21-25.

9. H. Soeder, Beiträge zur Funktionentheorie in Banachschen Räumen, Schriftenreihe Math. Inst. Univ. Münster, no. 9, 1956. 



\section{PACIFIC JOURNAL OF MATHEMATICS}

\section{EDITORS}

David GILbarg

Stanford University

Stanford, California

\section{F. H. BRowneLL}

University of Washington

Seattle 5, Washington

\section{A. L. Whiteman}

University of Southern California Los Angeles 7, California

\section{J. PAIGe}

University of California

Los Angeles 24, California

\section{ASSOCIATE EDITORS}
E. F. BECKENBACH
T. M. CHERRY
D. DERRY

\author{
E. HEWITT \\ A. HORN \\ L. NACHBIN
}

\author{
M. OHTSUKA \\ H. L. ROYDEN \\ M. M. SCHIFFER
}

E. SPANIER

E. G. STRAUS

F. WOLF

\section{SUPPORTING INSTITUTIONS}

\author{
UNIVERSITY OF BRITISH COLUMBIA \\ CALIFORNIA INSTITUTE OF TECHNOLOGY \\ UNIVERSITY OF CALIFORNIA \\ MONTANA STATE UNIVERSITY \\ UNIVERSITY OF NEVADA \\ NEW MEXICO STATE UNIVERSITY \\ OREGON STATE COLLEGE \\ UNIVERSITY OF OREGON \\ OSAKA UNIVERSITY \\ UNIVERSITY OF SOUTHERN CALIFORNIA
}

\author{
STANFORD UNIVERSITY \\ UNIVERSITY OF TOKYO \\ UNIVERSITY OF UTAH \\ WASHINGTON STATE COLLEGE \\ UNIVERSITY OF WASHINGTON \\ AMERICAN MATHEMATICAL SOCIETY \\ CALIFORNIA RESEARCH CORPORATION \\ HUGHES AIRCRAFT COMPANY \\ SPACE TECHNOLOGY LABORATORIES \\ NAVAL ORDNANCE TEST STATION
}

\footnotetext{
Mathematical papers intended for publication in the Pacific Journal of Mathematics should be typewritten (double spaced), and the author should keep a complete copy. Manuscripts may be sent to any one of the four editors. All other communications to the editors should be addressed to the managing editor, L. J. Paige at the University of California, Los Angeles 24, California.

50 reprints per author of each article are furnished free of charge; additional copies may be obtained at cost in multiples of 50 .
}

The Pacific Journal of Mathematics is published quarterly, in March, June, September, and December. The price per volume (4 numbers) is $\$ 12.00$; single issues, $\$ 3.50$. Back numbers are available. Special price to individual faculty members of supporting institutions and to individual members of the American Mathematical Society: $\$ 4.00$ per volume; single issues, $\$ 1.25$.

Subscriptions, orders for back numbers, and changes of address should be sent to Pacific Journal of Mathematics, 2120 Oxford Street, Berkeley 4, California.

Printed at Kokusai Bunken Insatsusha (International Academic Printing Co., Ltd.), No. 6, 2-chome, Fujimi-cho, Chiyoda-ku, Tokyo, Japan.

PUBLISHED BY PACIFIC JOURNAL OF MATHEMATICS, A NON-PROFIT CORPORATION

The Supporting Institutions listed above contribute to the cost of publication of this Journal, but they are not owners or publishers and have no responsibility for its content or policies. 


\section{Pacific Journal of Mathematics}

\section{Vol. 10, No. $4 \quad$ December, 1960}

M. Altman, An optimum cubically convergent iterative method of inverting a linear bounded operator in Hilbert space . . . . . . . . . . . . . . . . . . . . . . . . . . 1107

Nesmith Cornett Ankeny, Criterion for rth power residuacity ................. 1115

Julius Rubin Blum and David Lee Hanson, On invariant probability measures I . . . . . 1125

Frank Featherstone Bonsall, Positive operators compact in an auxiliary topology ..... 1131

Billy Joe Boyer, Summability of derived conjugate series . . . . . . . . . . . . . . . . 1139

Delmar L. Boyer, A note on a problem of Fuchs . . . . . . . . . . . . . . . . . 1147

Hans-Joachim Bremermann, The envelopes of holomorphy of tube domains in infinite

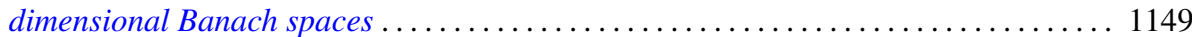

Andrew Michael Bruckner, Minimal superadditive extensions of superadditive

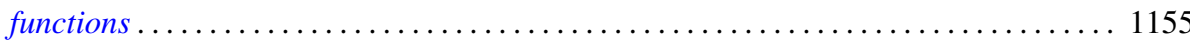

Billy Finney Bryant, On expansive homeomorphisms .................... 1163

Jean W. Butler, On complete and independent sets of operations in finite algebras . . . . . 1169

Lucien Le Cam, An approximation theorem for the Poisson binomial distribution ...... 1181

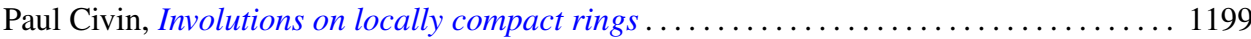

Earl A. Coddington, Normal extensions of formally normal operators . . . . . . . . . 1203

Jacob Feldman, Some classes of equivalent Gaussian processes on an interval ........ 1211

Shaul Foguel, Weak and strong convergence for Markov processes . . . . . . . . . . . 1221

Martin Fox, Some zero sum two-person games with moves in the unit interval ........ 1235

Robert Pertsch Gilbert, Singularities of three-dimensional harmonic functions . . . . . . . 1243

Branko Grünbaum, Partitions of mass-distributions and of convex bodies by

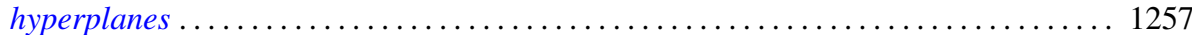

Sidney Morris Harmon, Regular covering surfaces of Riemann surfaces ........... 1263

Edwin Hewitt and Herbert S. Zuckerman, The multiplicative semigroup of integers

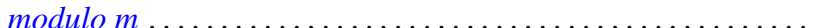

Paul Daniel Hill, Relation of a direct limit group to associated vector groups . ......... 1309

Calvin Virgil Holmes, Commutator groups of monomial groups . .

James Fredrik Jakobsen and W. R. Utz, The non-existence of expansive homeomorphisms

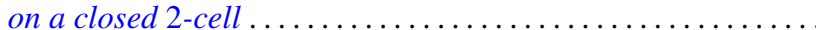

John William Jewett, Multiplication on classes of pseudo-analytic functions . . . . . . . 1323

Helmut Klingen, Analytic automorphisms of bounded symmetric complex domains . . . . 1327

Robert Jacob Koch, Ordered semigroups in partially ordered semigroups . . . . . . . . 1333

Marvin David Marcus and N. A. Khan, On a commutator result of Taussky and

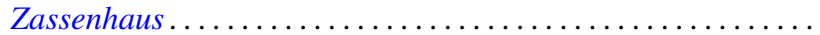

John Glen Marica and Steve Jerome Bryant, Unary algebras......

Edward Peter Merkes and W. T. Scott, On univalence of a continued fraction . . . . . . . 1361

Shu-Teh Chen Moy, Asymptotic properties of derivatives of stationary measures . . . . . 1371

John William Neuberger, Concerning boundary value problems . . . . . . . . . . . 1385

Edward C. Posner, Integral closure of differential rings . . . . . . . . . . . . . . . . . 1393

Marian Reichaw-Reichbach, Some theorems on mappings onto . . . . . . . . . . . . . 1397

Marvin Rosenblum and Harold Widom, Two extremal problems . . . . . . . . . . . . . . . . 1409

Morton Lincoln Slater and Herbert S. Wilf, A class of linear differential-difference

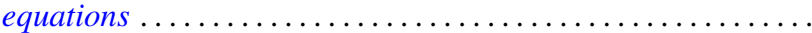

Charles Robson Storey, Jr., The structure of threads . . . . . . . . . . . . . . . . . . 1429

J. François Treves, An estimate for differential polynomials in $\partial / \partial z_{1},, \cdots, \partial / \partial z_{-} n \ldots \ldots 1447$

J. D. Weston, On the representation of operators by convolutions integrals . . . . . . . . 1453

James Victor Whittaker, Normal subgroups of some homeomorphism groups ......... 1469 